\title{
Metastatic intraocular hemangiopericytoma in a dog
}

\author{
Jonathan D. Pucket ${ }^{1, *}$, Rachel A. Allbaugh ${ }^{2}$, Mary L. Higginbotham ${ }^{3}$, Amy J. Rankin ${ }^{3}$ and Leandro Teixeira ${ }^{4}$ \\ ${ }^{1}$ Department of Veterinary Clinical Sciences, College of Veterinary Health Sciences, Oklahoma State University, \\ Stillwater, OK 74078, USA \\ ${ }^{2}$ Department of Veterinary Clinical Sciences, College of Veterinary Medicine, Iowa State University, Ames, IA \\ 50011, USA \\ ${ }^{3}$ Department of Clinical Science, College of Veterinary Medicine, Kansas State University, Manhattan, KS 66506, \\ USA \\ ${ }^{4}$ Department of Pathological Sciences, College of Veterinary Medicine, University of Wisconsin-Madison, WI \\ 53706, USA
}

\begin{abstract}
A 10-year-old Labrador Retriever who had been undergoing therapy for a recurrent hemangiopericytoma of the right flank presented to the Kansas State University Ophthalmology service for evaluation of a painful left eye. Examination revealed secondary glaucoma and irreversible blindness of the affected eye and multifocal chorioretinal lesions in the fellow eye. Therapeutic and diagnostic enucleation of the left eye was performed and histopathologic examination demonstrated the presence of a presumed metastatic spindle cell sarcoma. Further immunohistochemical staining confirmed the intraocular neoplasia to be metastatic spread from the previously removed flank mass. Rapid progression in size and number of chorioretinal lesions in the right eye was noted in the post-operative period until the patient was euthanized one month after surgery. This case report is the first to document intraocular metastasis of hemangiopericytoma in a veterinary patient.
\end{abstract}

Keywords: Glaucoma, Hemangiopericytoma, Metastasis.

\section{Introduction}

Malignant tumors can originate throughout the body, yet despite the well vascularized nature of the uveal tract metastasis to the ocular tissues is not a common occurrence. Lymphoma is the most common of those tumors which have been noted to spread to the eye (Hendrix, 2013). Less commonly documented intraocular metastatic neoplasms include melanoma, hemangiosarcoma, malignant histiocytosis, adenocarcinoma, osteosarcoma, transitional cell carcinoma, and others (Szymanski, 1972; Schmidt, 1981; Render et al., 1982; Szymanski et al., 1984; Habin and Elsa, 1995; Esson et al., 2007; Naranjo et al., 2007; Mowat et al., 2012).

Patients with metastatic ocular neoplasia may present with systemic signs attributable to the disease or may only have observable ophthalmic signs. Although possibly asymmetric in presentation, metastatic neoplasia is more likely to affect both eyes than primary tumors (Hendrix, 2013).

This case report is the first to describe metastatic spread of hemangiopericytoma to both eyes of a dog.

\section{Case Details}

A 10 year old, male neutered Labrador Retriever was referred to the ophthalmology service at Kansas State University's College of Veterinary Medicine due to left sided ocular discomfort, diffuse corneal edema, and possible vision loss. The patient was undergoing radiation therapy for a recurrent subcutaneous right flank mass, diagnosed as a hemangiopericytoma.

The original mass was removed from the right hip 18 months prior to presentation and on histopathology presented as a poorly delineated and infiltrative neoplastic tissue, partially effacing the superficial and deep dermis and composed of spindled to round cells with small amounts of pale or eosinophilic cytoplasm, indistinct cell borders and bland vesicular nuclei, arranged in streams and bundles forming variably ectatic or compressed, thin walled branching vascular channels.

Despite multiple surgical resections and complete excision according to histopathologic evaluation, the mass continued to recur subcutaneously in the right flank region. Based on the recurrence of the mass in the face of multiple resections, radiation therapy following excision and skin grafting was performed. Routine examination 4 months after radiation therapy showed a new subcutaneous mass in the right flank and multiple pulmonary nodules visible on computerized tomography (CT) scan. Two weeks after diagnosis of the pulmonary nodules the ocular signs became evident. 
Ophthalmic examination revealed slight blepharospasm and epiphora of the left eye (OS) along with an elevated third eyelid. No pain or significant adnexal abnormalities were noted in the right eye (OD) except for a single distichia dorsally. Menace response and dazzle reflexes were present OD but were both absent OS. Direct pupillary light response was present OD and absent OS. A consensual pupillary light response was not noted in either eye. Severe conjunctival hyperemia and moderate episcleral injection were noted OS. Fluorescein staining was negative in both eyes for epithelial defects. Intraocular pressures measured by rebound tonometry (TonoVet@; Jorgensen Labs, Loveland, CO) were 6 and $60 \mathrm{mmHg}$ OD and OS respectively.

On biomicroscopic examination (Kowa SL-15; Kowa Company, Tokyo, Japan) the cornea of the OS had moderate diffuse corneal edema making it difficult to visualize intraocular structures in detail, however, a dyscoric and relatively miotic pupil were detectable as well as a diffusely swollen and reddened iris profile. Extensive posterior synechia and iris swelling contributed to the dyscoria OS and structures posterior to the pupil could not be visualized. Examination of the cornea and anterior segment of the OD was unremarkable. Nuclear sclerosis was present OD as well as a ventromedial pinpoint anterior cortical incipient cataract and mild anterior vitreal syneresis. Upon indirect ophthalmoscopic examination (Vantage Plus Wireless, Keeler Instrument Inc, Bromall, PA) of the OD, multifocal lesions in the tapetal fundus were noted and photodocumented (RetCam Shuttle; Clarity Medical Systems, Pleasanton, CA) (Fig. 1A and 1B).
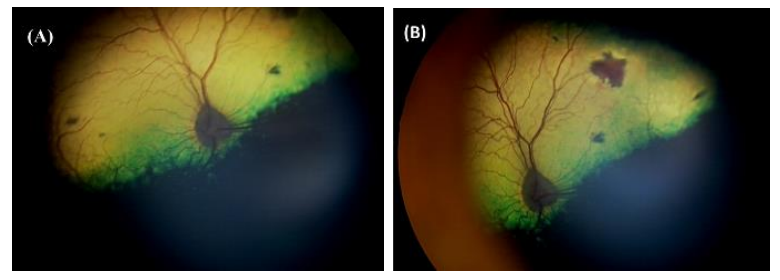

Fig. 1. Presence of chorioretinal lesions in the tapetal fundus OD noted on initial presentation. Images represent central view (A) and a view angled to highlight the largest chorioretinal lesion (B). Multifocal pink lesions are noted throughout the tapetal fundus arising from the termination of retinal vessels representing metastatic spread of the hemangiopericytoma. Images obtained with the RetCam Shuttle.

The largest lesion was raised, pale pink in color, one optic nerve head in diameter and located dorsomedial to the optic disc arising at the termination of a retinal vessel. Other smaller lesions appeared multifocally throughout the tapetal fundus as pinpoint reddish-pink colored lesions near the termination of retinal vessels, some of which could not be differentiated from retinal hemorrhages. Based on the clinical evidence, secondary glaucoma of the OS was diagnosed as well as suspected chorioretinal metastatic disease lesions OD.

Enucleation of the painful globe OS was recommended in order to provide comfort as well as diagnostic benefit through histopathology. Preoperative blood work was obtained and no significant abnormalities were noted. On physical examination, the patient was bright, alert and responsive as well as mildly overweight. No significant abnormalities were detected on thoracic auscultation or complete physical examination other than mild dental calculus, bilateral coxofemoral pain, and a $2 \times 3 \times 2 \mathrm{~cm}$ right flank subcutaneous mass. Fine needle aspirates of the subcutaneous mass performed five weeks prior revealed a spindle cell population with features of malignancy suggestive of a sarcoma. The left globe was removed using a transconjunctival approach and the patient recovered uneventfully. After removal, the globe was immersion fixed in $10 \%$ neutral buffered formalin and submitted to the Comparative Ocular Pathology Laboratory of Wisconsin for histopathologic evaluation.

Histopathology revealed a population of neoplastic spindle cells arranged in streams and bundles carpeting the surfaces of the iris and ciliary body, infiltrating and expanding the tapetal choroid and multifocally invading the choroidal vessels (Fig. 2A and 2B). The cells presented indistinct cell borders, small amounts of eosinophilic cytoplasm and oval to elongated nuclei with usually a large and central magenta nucleolus. Mitotic figures were common averaging 6 per high power field and cellular pleomorphism was marked with multiple karyomegalic cells. Neoplastic cells were also found to extend within scleral blood vessels (Fig. 2C). Notable secondary ocular lesions were posterior synechia, retinal detachment and secondary glaucoma, characterized by loss of ganglion cells and gliosis of the optic nerve head. The pattern of distribution of the neoplastic cells within the globe, with cells carpeting ocular surfaces and multifocally infiltrating the uveal tissue along with the presence of neoplastic cells in blood vessels was strongly suggestive of a metastatic disease. Sections of the previously excised flank hemangiopericytoma and the left globe were selected for immunohistochemistry to determine if the intraocular mass was metastatic or a separate neoplastic process. Sections were stained using the following antibodies: Skeletal muscle actin (mouse antisarcomeric actin, clone alpha-Sr-1, 1:150 dilution, Dako, Carpinteria, CA), Vimentin (mouse antivimentin, clone V9, 1:200, Dako), Alpha-smooth muscle actin (mouse anti-alpha smooth muscle actin, clone 1A4, 1:1000, Dako), S-100 (rabbit anti-S100 protein, 1:2500, Dako), CD31 (mouse anti-CD31, 1:40, Dako) and CD34 (mouse anti-CD34, 1:50, Dako). 

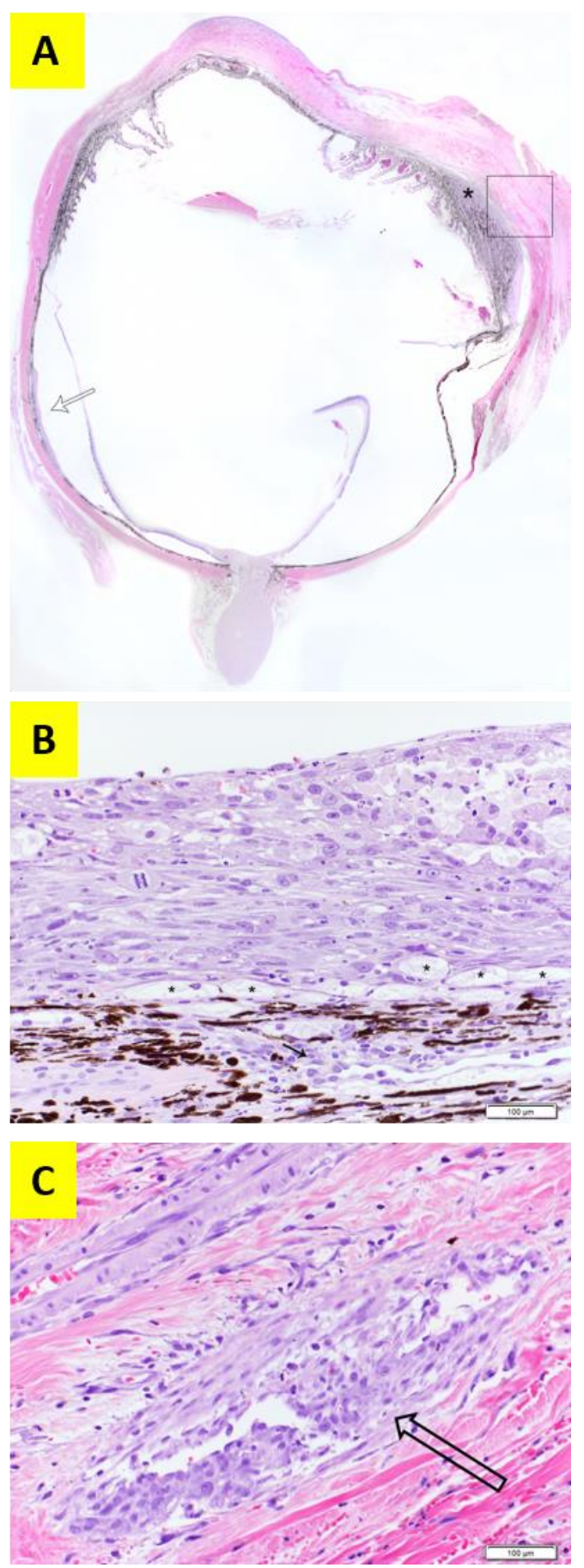

Fig. 2. (A): Metastatic spindle cell sarcoma. Subgross photograph. Note the carpet of neoplastic cells in the choroid (arrow) and in the ciliary body (*). Hematoxylin and eosin (H\&E). (B): Tumor in the choroid. Higher magnification of the neoplastic cells near the arrow in Fig. 2A. Cells infiltrate the tapetum (*) and surround choroidal vessels (arrow). $(\mathrm{H} \& \mathrm{E})(\mathrm{Bar}=100 \mu \mathrm{m}) .(\mathbf{C})$ : Tumor in the scleral vessels. Higher magnification of the square in Fig. 2A. Cells surround and infiltrate scleral vessels (arrow). $(\mathrm{H} \& \mathrm{E})$. $(\mathrm{Bar}=100 \mu \mathrm{m})$.
The results of the immunohistochemical staining showed perfect alignment in staining patterns in that the two separate masses were vimentin, alpha-smooth muscle actin and CD34 positive, while negative for skeletal muscle actin, S-100 and CD31 (Fig. 3). Together with the microscopic features of the flank mass (fusiform cells forming irregular vascular channels with a staghorn pattern) the immunohistochemical staining pattern confirmed the diagnosis of hemangioperycitoma and a metastatic spread of the right flank neoplasm to the eye in this case (Avallone et al., 2007).

The patient returned two weeks after surgery for routine enucleation site suture removal. Upon exam of the OD fluorescein staining was negative, the intraocular pressure was $5 \mathrm{mmHg}$, the direct pupillary light reflex was positive and a menace response was present. Despite the lower intraocular pressure, there was no evidence of blepharospasm, epiphora, conjunctival hyperemia, miosis, or aqueous flare on her exam.

No changes in the lenticular opacities were observed, however, fundic examination revealed that the chorioretinal lesions had progressed significantly in size and number throughout the tapetal fundus (Fig. 4). Perilesional retinal detachments were identified around the larger pink colored lesions. Despite disease progression, the patient remained comfortable and visual. Prophylactic topical anti-inflammatory and topical anti-glaucoma therapy were discussed with the owner given the previous progression OD and previous sequelae OS but were declined. Five days after suture removal the patient presented for acute blepharospasm and epiphora of the OD. Fluorescein staining was negative and the intraocular pressure was $6 \mathrm{mmHg}$. A small fibrin clot was noted on the anterior surface of the lens capsule axially along with a trace amount of aqueous flare. No significant progression of the chorioretinal lesions was detected. The patient was started on $1 \%$ topical ophthalmic prednisolone acetate suspension (Falcon Pharmaceuticals, Hünenberg, Switzerland) every 8 hours as well as $2.2 \mathrm{mg} / \mathrm{kg}$ of oral carprofen (Rimadyl; Pfizer Inc, New York, NY) every twelve hours. In addition, despite a low but seemingly normal intraocular pressure, topical $2 \%$ dorzolamide (Dorzolamide HCL 2\% ophthalmic solution; Hi-Tech Pharmacal Co, Amityville, NY) solution was instituted every 8 hours OD given previous progression to secondary glaucoma OS. A recheck was scheduled for 1 week. Three days later the patient presented on emergency for difficulty urinating and a gallop rhythm was ausculted on physical exam. Radiographs showed multiple small cystic and urethral calculi, slight enlargement of the previously noted pulmonary nodules, and right sided cardiac enlargement. 


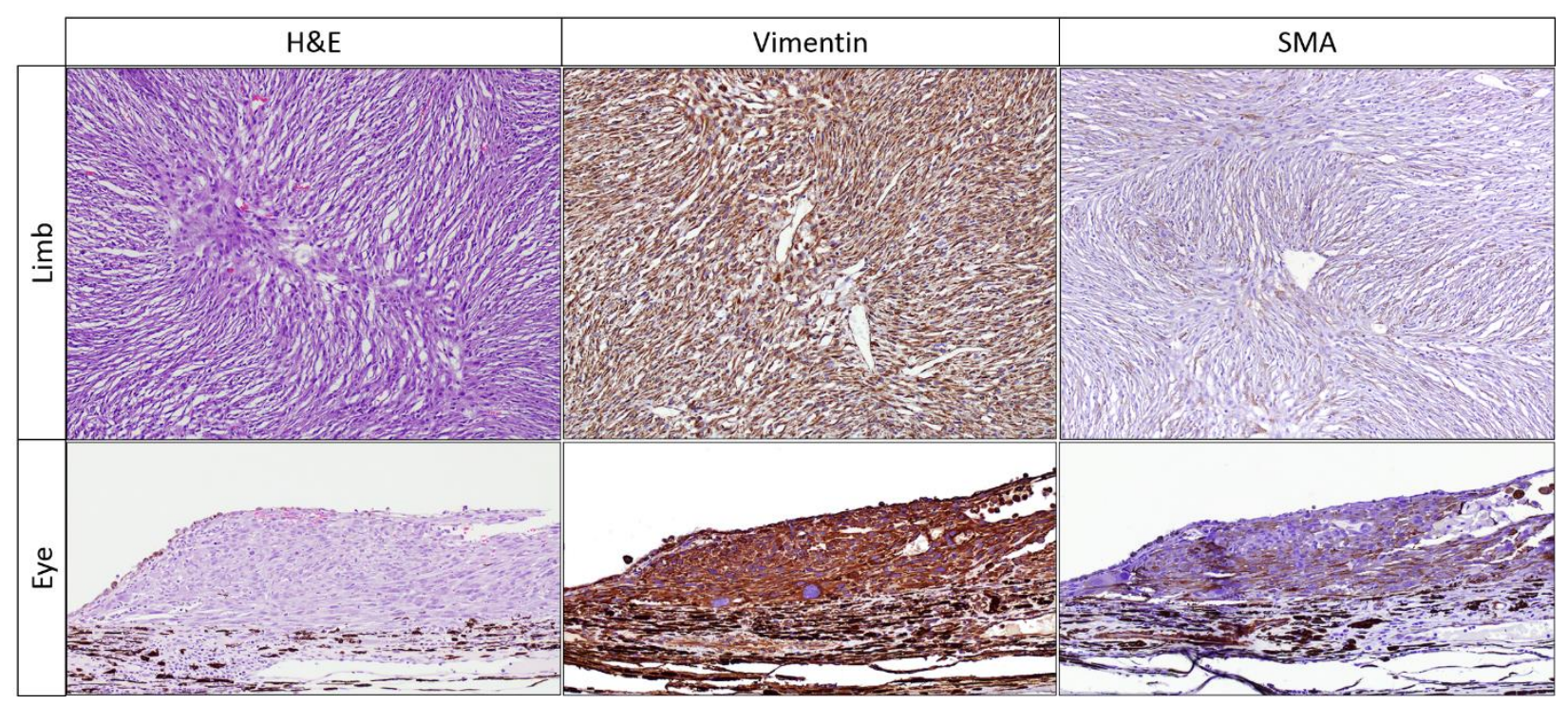

Fig. 3. Immunohistochemical staining of both the original right flank/limb hemangiopericytoma and the left eye metastasis. Both tissues show positive staining for vimentin and alpha-smooth muscle actin (SMA).

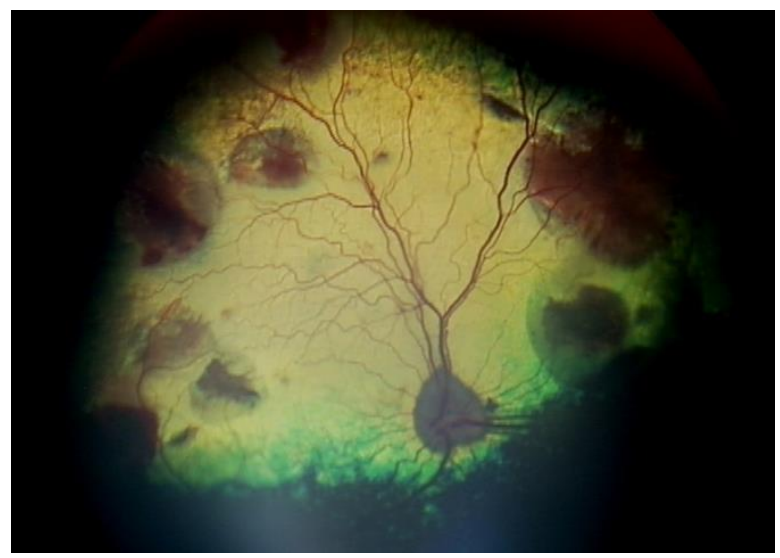

Fig. 4. Image of right tapetal fundus two weeks after initial presentation. The presence of numerous large pink/red colored lesions throughout tapetal fundus present near retinal vessel terminations showing significant progression of chorioretinal lesions. Perilesional retinal elevations are seen around each foci of metastasis and appear as halos of reduced reflectivity. Image obtained with the RetCam Shuttle.

On echocardiogram, a $5 \times 3 \mathrm{~cm}$ heteroechoic mass was noted arising from the right atrium and extending into the auricle with moderate pericardial effusion but no cardiac tamponade. Due to the extensive nature and poor prognosis, the patient had a urethral catheter placed and was discharged for palliative care by the owner and referring veterinarian. One week later the patient was euthanized. A postmortem examination was not performed and no tissues were available for evaluation.

\section{Discussion}

Perivascular wall tumor is a term used to describe the grouping of soft tissue sarcomas which have origins in the structural and supportive cells of blood vessels (Avallone et al., 2007).

Hemangiopericytoma refers to a sub-category of perivascular wall tumors and is a rare vascular tumor that is thought to arise from mesenchymal cells which coat capillaries and post capillary venules, known as pericytes of Zimmerman. This type of tumor was first described by Stout and Murray (1942).

Since the initial manuscript there have been numerous reports documenting this tumor in humans and various veterinary species (Mulligan, 1955; Enzinger and Smith, 1976; Richardson et al., 1983; Boniuk et al., 1985; Fossum et al., 1988; Graves et al., 1988; Mitarai et al., 1998; Beltran et al., 2001; McCaw et al., 2001; Serena et al., 2006; Silva et al., 2014).

Most commonly, hemangiopericytoma is reported in dogs, of which it accounts for around 3-4\% of all cutaneous neoplasia (Graves et al., 1988). The tumors are reported to be well circumscribed initially with a pseudocapsule covering the mass (Henderson and Farrow, 1978; Sujatha et al., 1994) and attached to deeper structures with freely movable skin over the surface (Caniatti et al., 2001). If the pseudocapsule is breached during surgery or incomplete excision is noted, extensive local regrowth and metastasis can be seen (Shimura et al., 2001).

Proper diagnosis of hemangiopericytomas can be challenging from a histopathologic standpoint and recent investigations show that many previous cases diagnosed as such may actually represent one of a spectrum of perivascular wall tumors (Avallone et al., 2007; Palmieri et al., 2013). The originally described microscopic pattern of a hemangiopericytoma consisted of concentric layers of spindle cells arranged 
around a central vessel which was devoid of erythrocytes, known as a "fingerprint, staghorn, or onion skin pattern" (Perez et al., 1996). This pattern is often lacking and non-specific as it can even be found in other types of vascularized soft-tissue neoplasms (Pantekoek and Schiefer, 1975).

Definitive diagnosis requires immunohistochemical staining or electron microscopy to differentiate hemangiopericytoma from other soft tissue sarcomas (Avallone et al., 2007; Palmieri et al., 2013). Previous reports have tried to elucidate the immunoreactivity patterns to aide differentiation. Hemangiopericytoma cells stain positive for vimentin and CD 34, owing to their mesenchymal and endothelial cell derivation respectively (Perez et al., 1996; Middleton et al., 1998). Staining for S-100 and CD 31 are negative, proving the neoplasms are not of peripheral nerve, melanocyte, granular cell, macrophage, or monocyte origin (Middleton et al., 1998). Staining for muscle actins can be variable depending on their location and if describing human or canine hemangiopericytoma. While canine hemangiopericytomas express muscle actins, human hemangiopericytomas are lacking this trait. This means that the human neoplasia counterpart either loses the expression during differentiation, or the cell of origin is not a true pericyte (Porter et al., 1991; Perez et al., 1996).

In dogs, the expression or not of $\alpha$-smooth muscle actin depends on whether the tumor formed from capillary or arteriolar and venular pericytes (Herman and D'amore, 1985; Schürch et al., 1987). Alpha-smooth muscle actin is expressed in hemangiopericytomas that arise from arteriolar or venular pericytes and non-smooth muscle actin isoforms are derived from those of capillary origin (Herman and D'amore, 1985). This explains the variable staining noted in hemangiopericytomas with regard to $\alpha$-smooth muscle actin. In our case, both the original flank mass and the intraocular metastasis lesions were in perfect alignment with regard to reported hemangiopericytoma immunohistochemical staining patterns, confirming the diagnosis.

Hemangiopericytomas tend to develop on distal extremities of older dogs and have a slow growth rate (Mulligan, 1955; Graves et al., 1988; Mazzei et al., 2002).

In humans, common locations for development of hemangiopericytoma are the thigh and pelvic retroperitoneal regions (Enzinger and Smith, 1976; Lee et al., 2003). Average age at the time masses are observed in dogs is around 10 years, with a range of 214 years (Graves et al., 1988; Caniatti et al., 2001; Namazi et al., 2014).

The frequency of metastasis is presumed to be low based on available cases, but local recurrence is high due to the infiltrative nature of the mass (Yost and Jones, 1958; Handharyani et al., 1999). Recurrence rates after excision approximate $40 \%$ even if all grossly visible tumor has been removed (Graves et al., 1988). A statistically significant difference has not been shown between simple excision, excision with orthovoltage radiation, and excision with subsequent photodynamic therapy (Graves et al., 1988; McCaw et al., 2001).

Seven previous canine cases have been recognized to have metastasized, and of those the lungs and lymph nodes were sites of spread (Handharyani et al., 1999; Silva et al., 2014). In humans, the rate of metastasis has been reported to be as high as $50 \%$, with spread to the lungs, liver, and bones occurring most frequently (Johnson, 1976). The reason for the low rate of metastasis in dogs as compared to humans has not been determined.

In humans, hemangiopericytoma is very rarely found in or around the eye with the majority of cases being retrobulbar in origin (Henderson and Farrow, 1978; Karcioglu et al., 1997; Lee et al., 2003; Manjandavida et al., 2013). Other reported locations include the lacrimal sac, optic nerve, conjunctiva, and eyelids (Boniuk et al., 1985; Grossniklaus et al., 1986; Sujatha et al., 1994; Charles et al., 1998; Parmar and Rose, 2003; Schwent et al., 2007). There have been only 5 cases of intraocular hemangiopericytoma reported to date (Papale et al., 1983; Gieser et al., 1988; Brown et al., 1991; Toth et al., 1996; Shimura et al., 2001). Of these most were considered primary lesions, however, metastatic spread from another primary location was not completely ruled out.

In veterinary patients, the reported incidence of ocular hemangiopericytoma is also low with only 2 reported cases. The first was a presumed orbital hemangiopericytoma in a seven year old mixed-breed dog presenting with progressive unilateral exophthalmos (Beltran et al., 2001). The tumor was diagnosed by histopathologic evaluation after removal, however immunohistochemical staining was not performed to differentiate from peripheral nerve sheath tumors or other soft tissue sarcomas. The other ocular hemangiopericytoma was documented in an eyelid of a fourteen year old Arabian horse (Serena et al., 2006). The mass had been present for two years but then rapidly increased in size, prompting exam and removal. The diagnosis was confirmed through immunohistochemical staining of excised tissue. One year after removal, no recurrence of the mass or spread was noted.

To the authors' knowledge, the case reported here is the first to document presumed intraocular metastatic spread of a perivascular wall tumor in a veterinary patient.

Unlike previously reported ocular manifestations, the hemangiopericytoma in our case progressed rapidly. The findings of a right sided heart mass and the suspected pulmonary metastatic lesions during the 
course of diagnosis and therapy are interesting but the significance is not known as diagnostic samples were never obtained.

In addition, a post mortem examination was not performed and so any attempt to correlate these to the primary hemangiopericytoma would be unfounded and only the ocular metastasis lesions were confirmed. Intraocular metastasis may represent a late stage of progression with hemangiopericytoma or could signal a more aggressive tumor as evidenced by the very short survival time of this patient after recognized intraocular spread.

\section{Conflict of Interest}

The Authors declare no conflict of interest.

\section{References}

Avallone, G., Helmbold, P., Caniatti, M., Stefanello, D., Nayak, R. and Roccabianca, P. 2007. The spectrum of canine cutaneous perivascular wall tumors: morphologic, phenotypic and clinical characterization. Vet. Pathol. 44, 607-620.

Beltran, W.A., Colle, M., Boulouha, L., DaudeLagrave, A., Moissonnier, P. and Clerc, B. 2001. A case of orbital hemangiopericytoma in a dog. Vet. Ophthalmol. 4, 255-259.

Boniuk, M., Messmer, E. and Font, R. 1985. Hemangiopericytoma of the meninges of the optic nerve. A clinicopathologic report including electron microscopic observations. Ophthalmol. 92, 17801787.

Brown, H., Brodsky, M., Hembree, K. and Mrak, R. 1991. Supraciliary hemangiopericytoma. Ophthalmol. 98, 378-382.

Caniatti, M., Ghisleni, G., Ceruti, R., Roccabianca, P. and Scanziani, E. 2001. Cytological features of canine haemangiopericytoma in fine needle aspiration biopsy. Vet. Rec. 149, 242-244.

Charles, N., Palu, R. and Jagirdar, J. 1998. Hemangiopericytoma of the lacrimal sac. Arch. Ophthalmol. 116, 1677-1680.

Enzinger, F. and Smith, B 1976. Hemangiopericytoma. An analysis of 106 cases. Hum. Pathol. 7, 61-82.

Esson, D., Fahrer, C., Zarfoss, M. and Dubielzig, R. 2007. Suspected uveal metastasis of a nail bed melanoma in a dog. Vet. Ophthalmol. 10, 262-266.

Fossum, T., Couto, C., DeHoff, W. and Smeak, D. 1988. Treatment of hemangiopericytoma in a dog using surgical excision, radiation, and a thoracic pedicle skin graft. J. Am. Vet. Med. Assoc. 193, 1440-1442.

Gieser, S., Hufnagel, T., Jaros, P., MacRae, D. and Khodadoust, A. 1988. Hemangiopericytoma of the ciliary body. Arch. Ophthalmol. 106, 1269-1272.

Graves, G., Bjorling, D. and Mahaffey, E. 1988. Canine hemangiopericytoma: 23 cases (1967-1984). J. Am. Vet. Med. Assoc. 192, 99-102.
Grossniklaus, H., Green, W., Wolff, S. and Iliff, N. 1986. Hemangiopericytoma of the conjunctiva. Two cases. Ophthalmol. 93, 265-267.

Habin, D. and Else, R. 1995. Parotid salivary gland adenocarcinoma with bilateral ocular and osseous metastases in a dog. J Small Anim Pract 36, 445449.

Handharyani, E., Ochiai, K, Kadosawa, T., Kimura, T. and Umemura, T. 1999. Canine hemangiopericytoma: an evaluation of metastatic potential. J. Vet. Diag. Invest. 11, 474-478.

Henderson, J. and Farrow, G. 1978. Primary orbital hemangiopericytoma. An aggressive and potentially malignant neoplasm. Arch. Ophthalmol. 96, 666-673.

Hendrix, D. 2013. Diseases and surgery of the canine anterior uvea. Veterinary Ophthalmology. Gelatt, K.N., B.C. Gilger and T.J. Kern. Ames, IA, John Wiley \& Sons, pp: 1146-1198.

Herman, I. and D'amore, P. 1985. Microvascular pericytes contain muscle and nonmuscle actins. J. Cell Biol. 101, 43-52.

Johnson, C. 1976. Pathology of cutaneous vascular tumors. Int. J. Dermatol. 15, 256-259.

Karcioglu, Z., Nasr, A. and Haik, B. 1997. Orbital hemangiopericytoma: clinical and morphologic features. Am. J. Ophthalmol. 124, 661-672.

Lee, Y., Wang, J. and Shyu, J. 2003. Orbital hemangiopericytoma-a case report. The Kaohsiung J. Med. Sci. 19, 33-37.

Manjandavida, F., Honavar, S., Gowrishankar, S., Mulay, K., Reddy, V. and Vemuganti, G. 2013. Optic nerve meningeal hemangiopericytoma: a clinicopathologic case report. Surv. Ophthalmol. 58, 341-347.

Mazzei, M., Millanta, F., Citi, S., Lorenzi, D. and Poli, A. 2002. Hemangiopericytoma: histological spectrum, immunohistochemical characterization and prognosis. Vet. Dermatol. 13, 15-21.

McCaw, D., Payne, J., Pope, E., West, M., Tompson, R. and Tate, D. 2001. Treatment of canine hemangiopericytomas with photodynamic therapy. Lasers Surg. Med. 29, 23-26.

Middleton, L., Duray, P. and Merino, M. 1998. The histological spectrum of hemangiopericytoma: application of immunohistochemical analysis including proliferative markers to facilitate diagnosis and predict prognosis. Hum. Pathol. 29, 636-640.

Mitarai, Y., Ishikawa, Y. and Kadota, K. 1998. Hemangiopericytoma in a calf. Res. Vet. Sci. 65, 265-267.

Mowat, F., Langohr, I., Bilyk, O., Koterbay, A., Pierce, K. and Petersen-Jones, S. 2012. Bilateral uveal metastasis of a subcutaneous fibrosarcoma in a cat. Vet. Ophthalmol. 15, 391-397. 
Mulligan, R. M. 1955. Hemangiopericytoma in the dog. Am. J. Pathol. 31, 773-789.

Namazi, F., Abbaszadeh Hasiri, M., Oryan, A. and Moshiri, A. 2014. Hemangiopericytoma in a young dog: Evaluation of histopathological and immunohistochemical features. Vet. Res. Forum 5, 157-160.

Naranjo, C., Dubielzig, R. and Friedrichs, K. 2007. Canine ocular histiocytic sarcoma. Vet. Ophthalmol. 10, 179-185.

Palmieri, C., Avallone, G., Cimini, M., Roccabianca, P., Stefanello, D. and Salda, L. 2013. Use of electron microscopy to classify canine perivascular wall tumors. Vet. Pathol. 50, 226-233.

Pantekoek, J. and Schiefer, B. 1975. Metastasising canine fibrosarcoma originally diagnosed as haemangiopericytoma. J. Small Anim. Pract. 16, 259-265.

Papale, J., Frederick, A. and Albert, D. 1983. Intraocular hemangiopericytoma. Arch. Ophthalmol. 101, 1409-1411.

Parmar, D. and Rose, G. 2003. Management of lacrimal sac tumours. Eye (London) 17, 599-606.

Perez, J., Bautista, M., Rollón, E., de Lara, F., Carrasco, L. and Martin de las Mulas, J. 1996. Immunohistochemical characterization of hemangiopericytomas and other spindle cell tumors in the dog. Vet. Pathol. 33, 391-397.

Porter, P., Bigler, S., McNutt, M. and Gown, A. 1991. The immunophenotype of hemangiopericytomas and glomus tumors, with special reference to muscle protein expression: an immunohistochemical study and review of the literature. Mod. Pathol. 4, 46-52.

Render, J., Carlton, W., Vestre, W. and Hoerr, F. 1982. Osteosarcoma metastatic to the globes in a dog. Vet. Pathol. 19, 323-326.

Richardson, R., Render, J., Rudd, R., Shupe, R. and Carlton, W. 1983. Metastatic canine hemangiopericytoma. J. Am. Vet. Med. Assoc. 182, 705-706.
Schmidt, R.E. 1981. Transitional cell carcinoma metastatic to the eye of a dog. Vet. Pathol. 18, 832834.

Schürch, W., Skalli, O., Seemayer, T. and Gabbiani, G. 1987. Intermediate filament proteins and actin isoforms as markers for soft tissue tumor differentiation and origin. I. Smooth muscle tumors. Am. J. Pathol. 128, 91.

Schwent, B., Wojno, T. and Grossniklaus, H. 2007. Hemangiopericytoma of the optic nerve sheath. Am. J. Ophthalmol. 143, 904-906.

Serena, A., Joiner, K. and Schumacher, J. 2006. Hemangiopericytoma in the eyelid of a horse. Vet. Pathol. 43, 576-578.

Shimura, M., Suzuki, K., Fuse, N., Yoshida, M., Saiki, Y., Ohtani, H. and Tamai, M. 2001. Intraocular hemangiopericytoma. A case report. Ophthalmologica 215, 378-382.

Silva, E., Romero, F., Green, K., Martins, M. and Bracarense, A. 2014. Hemangiopericytoma in a female dog with direct invasion of abdominal cavity and pulmonary metastasis. Ciênc 44, 358-361.

Stout, A. and Murray, M. 1942. Hemangiopericytoma: a vascular tumor featuring Zimmerman's pericytes. Ann. Surg. 116, 22-33.

Sujatha, S., Sampath, R., Bonshek, R. and Tullo, A. 1994. Conjunctival haemangiopericytoma. Br. J. Ophthalmol. 78, 497-499.

Szymanski, C., Boyce, R. and Wyman, M. 1984. Transitional cell carcinoma of the urethra metastatic to the eyes in a dog. J. Am. Vet. Med. Assoc. 185, 1003-1004.

Szymanski, C.M. 1972. Bilateral metastatic intraocular hemangiosarcoma in a dog. J. Am. Vet. Med. Assoc. 161, 803-805.

Toth, J., Kerenyi, A., Suveges, I. and Futo, G. 1996. Leiomyoma of the ciliary body and hemangiopericytoma of the choroid. Pathol. Oncol. Res. 2, 89-93.

Yost, D. and Jones, T. 1958. Hemangiopericytoma in the dog. Am. J. Vet. Res. 19, 159-163. 